\title{
Design of the Storage Battery Online Monitoring System
}

\author{
Juan Tian \\ College of Information Engineering, Taishan Medical University, Tai'an 271016, China
}

Cheng Wang

Shandong Senter Electronic Co., Ltd, Zibo 255000, China

Xixue Zhang

College of Information Engineering, Taishan Medical University, Tai'an 271016, China

\begin{abstract}
Storage battery is the integrant back power supply in the communication system, and its using life and security reliability are specially concerned by users. To avoid the accidents induced by the individual battery failure in the long-term using process, the running status of the storage battery must be maintained and monitored periodically. A new and advanced capacity measurement technology, i.e. the whole-online discharge monitoring technology is proposed in this article. Comparing with other discharge technologies, it could utilize the existing real loads and the locale power cut to complete the data acquisition of the storage battery without any adjustment and special power cut, and it has no any risk to realize the online monitoring and in-time failure diagnose to the storage battery, and it could not only simplify the battery discharge maintenance operation, but enhance the working efficiency of the maintenance.
\end{abstract}

Keywords: Storage battery, Monitoring system, Whole-online discharge technology, Visual Basic 6.0

\section{Introduction}

The power supply of communication is the base of the communication network, and the storage battery is the final security barrier of the whole communication power supply. To ensure the reliability of the communication power supply, the capacity and the working status of the storage battery must be known in time. Through the analysis of the accidents in the communication system in recent years, in the communication interrupts induced by the power supply, the accidents induced by the storage battery occupied above $70 \%$. Once the accident of the storage battery, especially the storage battery in the communication room happens, large loss will occur. Therefore, in the practice, it is very important to study the problem how to reasonably and reliably manage and maintain the storage battery.

\section{Hardware design of the system}

A new and advanced capacity measurement technology, i.e. the whole-online discharge technology, is introduced in this article. It could realize the real-time online monitoring and the in-time failure diagnose of the storage battery, and complete the data acquisition of the storage battery. The new technology could acquire the charge and discharge data of each single cell real time by the charge and discharge test of the actual load of the measured pile, and the acquired data are transmitted to the background management software to plot the real-time change curve of each single cell, so the performance of each single cell could be judged according to the change curve. This technology needs not cutting the single cell, and it could complete the single cell management analysis of the whole storage pile by existing single cell data acquisition equipment and existing loading, which could largely enhance the efficiency, reduce the risk, and achieve the maintenance effect of safety and energy-saving. The structure figure is seen in Figure 1.

The hardware part of this system includes two modules, i.e. the upper computer and the intelligent measurement module of the storage battery.

The upper computer is linked with the intelligent measurement module of the storage battery by the RS-485 which receives the information and data. Its main function is to realize the series communication function of the intelligent measurement module by the VB programming software, and more visually display the total voltage of the storage battery, the electric current of charge and discharge, the voltage of the single cell, and store the voltage of each single storage battery into the database for the comparison of various present single voltage and the measurement of the change of each single battery, so the performance of the battery can be judged, and users could pay attention to special storage battery and refresh special storage battery in time. 
The intelligent measurement module of the storage battery is the data acquisition module of the storage battery in essential, which could not only collect and store the total voltage of the storage battery, the voltage of single battery, the current of charge and discharge, and the temperature of the storage battery, but also display the collected data on the upper computer, and the structure frame is seen in Figure 2.

The intelligent measurement module of the storage battery is composed by the SCM, the online measurement circuit, the address setting circuit, the temperature measurement circuit, the communication circuit, and the power supply circuit.

\section{$2.1 S C M$}

SCM is the core of the system. The SCM in this system is C8051F020 SCM made by Cygnal Company. And it is a strengthened 51 kernel SCM, and its order system is completely compatible with MCS-51, and it includes 64KB FLASH program memorizer, 4352B RAM, 8 I/O ends, one 12-bit A/D converter, 5 16-bit general timer, watchdog timer. Comparing with former 51 series SCM, C8051F020 has many new functions, and its reliability and speed are also enhanced largely.

\subsection{Online measurement circuit}

The storage battery in this article is connected by $242 \mathrm{~V}$ cells in series, and the voltage acquisition circuit is designed specially. The electrical isolation circuit designed in this article is realized by the circuit composed by the optical coupler AQW214 and high-precision capacitances. The working principle is seen in Figure 3. RL1 and RL2 are Pelay-DPDT (optical coupler Relay AQW214), and in the state of ready mode, the switch is on the side of Vin to charge the capacitance $\mathrm{C} 1$, and when measuring the voltage of the battery, the switch is put to the side of Vout, and returns to the side of Vin after the measurement ends. If the impedance of load R1 is infinite, because of the storage function of $\mathrm{C} 1$, Vout equals to Vin before switching, so the isolation switching of the measurement is completed.

\subsection{Temperature measurement circuit}

The temperature measurement circuit designed in this article is composed by 3 DS18B20 temperature sensors of US Dallas Semiconductor Company. DS18B20 temperature sensor is a kind of single-bus temperature sensor with many advantages such as small volume, high precision, and wide voltage range, and it sues the on-board temperature measurement patent technology to support the interface of "one-line bus", and the spot temperature is transmitted by the digital mode of "one-line bus" directly, which could largely enhance the anti-jamming performance of the system, and acquire good temperature measurement effect in practice.

Each DS18B20 can be set as two modes of power supply, i.e. the exterior power supply mode and the data bus power supply mode. In this design, the exterior power supply mode is selected to supply power to the DS18B20, so the circuit link is simpler and more flexible.

In addition, the address setting circuit adopts 8-bit address dial switch which could set up 256 different addresses.

\section{Software design of the system}

The software design of the whole system includes two parts, i.e. the software design of the intelligent measurement module and the software design of the upper computer.

\subsection{Software design of the intelligent measurement module}

The software of the intelligent measurement module is compiled by Keil C51, and the design process of the program includes three layers. The first layer is the design of the basic operation layer including the setting and operation of the register and the terminal operation. The second layer is the design of the driver layer including the basic driver of various peripherals such as the bus driver of DS18B20. The third layer is the design of the application layer including concrete applications such as the encapsulation and resolution of the communication protocol.

To enhance the readability and the portability of program, the main functions of program all adopt the modeling design methods, and there are six modules, i.e. the system setting and the initialization program, the address setting reading drive program, the $\mathrm{AD}$ conversion driver program, the temperature sensor one-line bus driver program, the communication driver program, and the application program (including the disposal of the address setting, the single cell voltage, the total voltage, the disposal of the total current, the disposal of the temperature, the encapsulation and resolution of the communication protocol). And the system setting and initialization program flow is seen in Figure 4. 


\subsection{Software design of the upper computer}

The upper computer software development is completed by the Visual Basic 6.0 which could not only dynamically display the data collected by the intelligent measurement module real time, but realize the storage and inquiry of the historical data. Not only this monitoring system has friendly user interface, but also it is more flexible and efficient. The interface figure is seen in Figure 5.

\section{Conclusions}

A new and advanced capacity measurement technology, i.e. the whole-online discharge monitoring technology is proposed in this article. Comparing with other discharge technologies, it could utilize the existing real loads and the locale power cut to complete the data acquisition of the storage battery without any adjustment and special power cut, and it has no any risk to realize the online monitoring and in-time failure diagnose to the storage battery, and it could not only simplify the battery discharge maintenance operation, but enhance the working efficiency of the maintenance. Based on that, the intelligent measurement module of the storage battery is designed including the hardware and the software, and it could not only measure the total voltage of the storage battery, the voltage of single battery, the current of charge and discharge, and the temperature of the storage battery, but also display the collected data on the upper computer through the series communication RS- 485 .

\section{References}

Ji, Aihua, Zhou, Zhimin \& Zhou, Jihai. (2004). Practical Technology of Valve Regulated Sealed Lead-acid Battery. Beijing: China Power Press.

Li, Liwei. (2006). Theoretical and Application Research of the DC System Failure Monitoring. Dalian: Doctor's Degree Thesis of Dalian University of Technology.

Li, Liwei \& Zou, Jiyan. (2003). Research on the Internal Resistance Measuring Device for Battery. Chinese Journal of Power Sources. No.1.

Mei, Lifeng. (2004). SCM Principle and Interface Technology. Beijing: Tsinghua University Press.

Xu, Manzhen. (2005). Principle and Application of New Storage Battery. Beijing: Posts \& Telecom Press.

Zhang, Honglin et al. (1999). Visual Basic 6.0 Development Database. Beijing: Posts \& Telecom Press.

Zhou, Cihang. (2002). SCM Application Program Design Technology. Beijing: Beijing University of Aeronautics and Astro-nautics Press. 


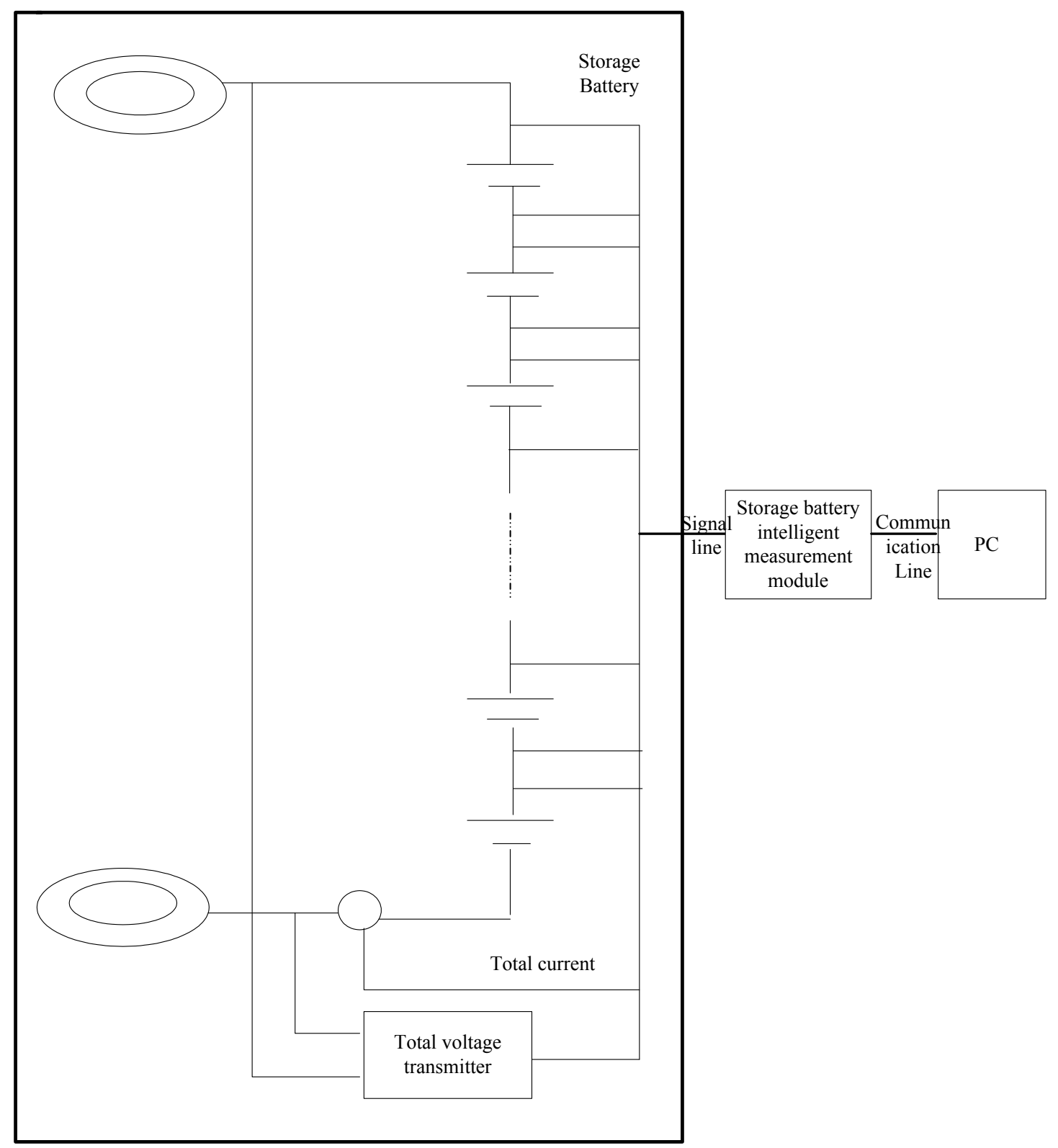

Figure 1. Structure of the Storage Battery Online Monitoring System 


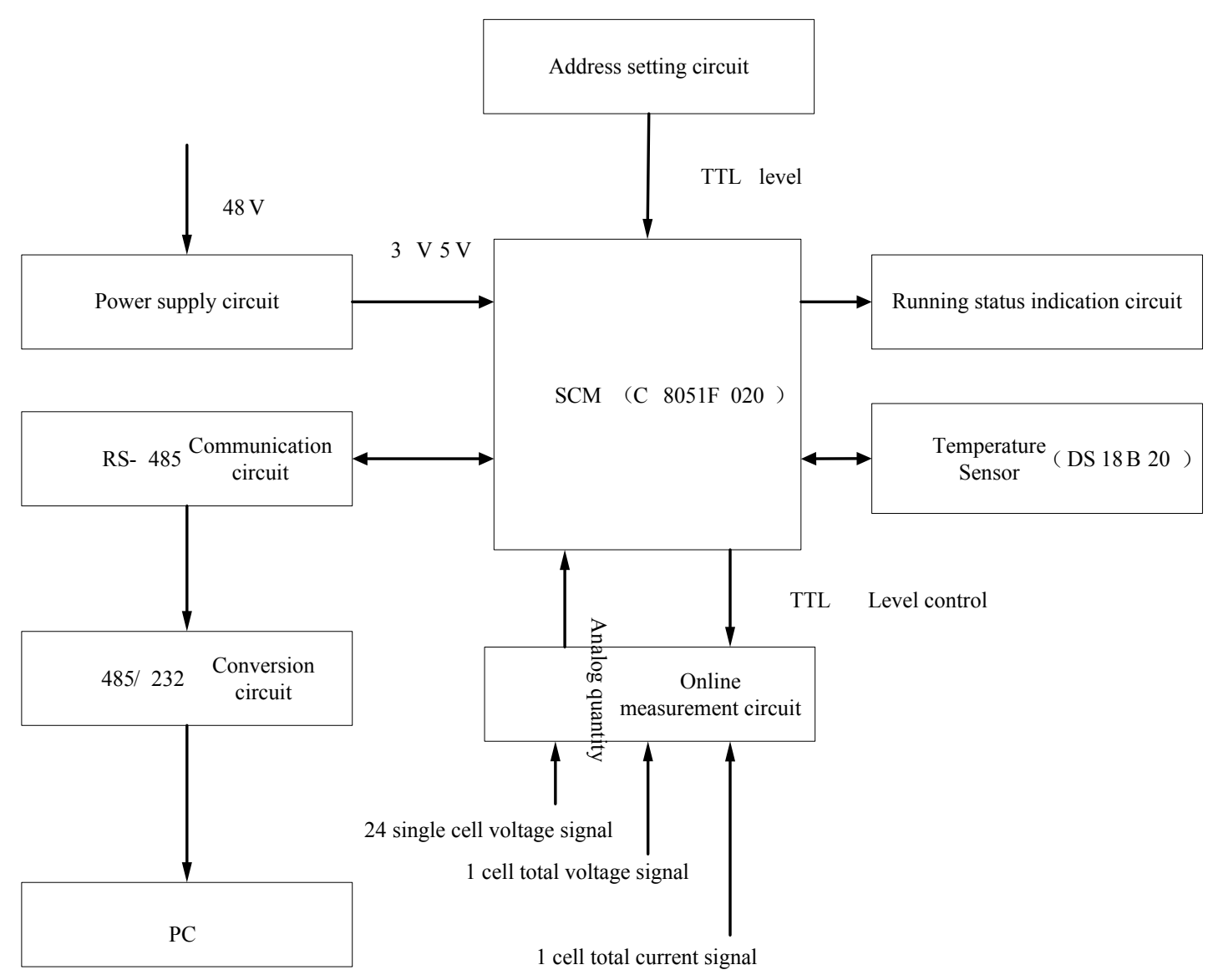

Figure 2. Structure of the Storage Battery Intelligent Monitoring Module

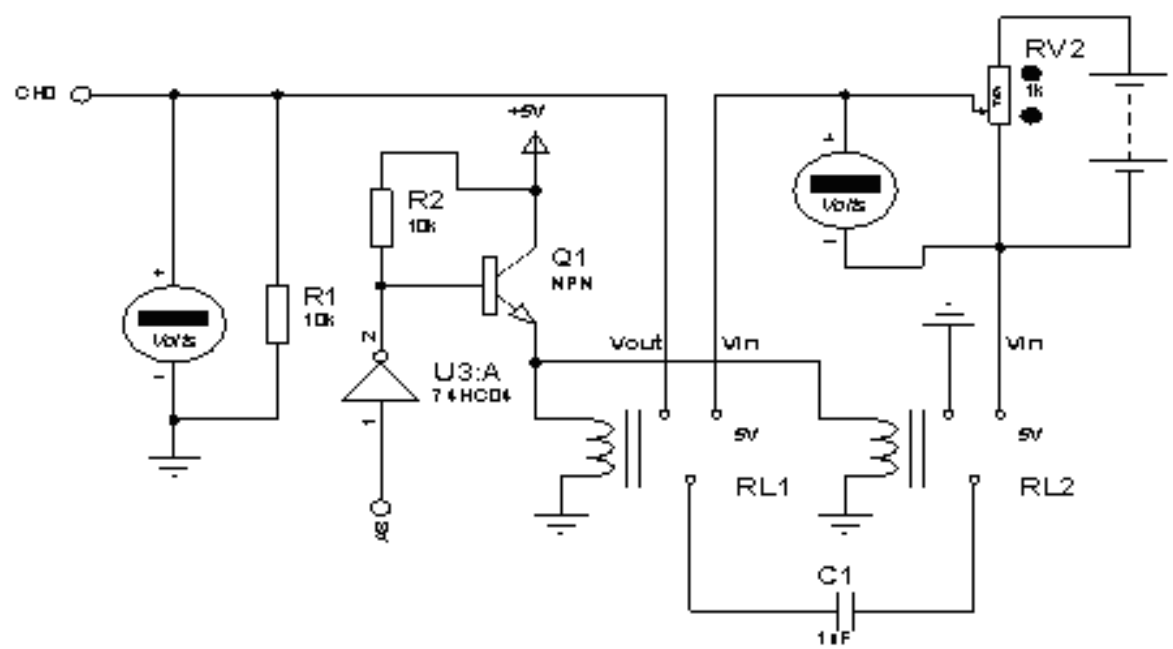

Figure 3. Working Principle of the Online Measurement Circuit 


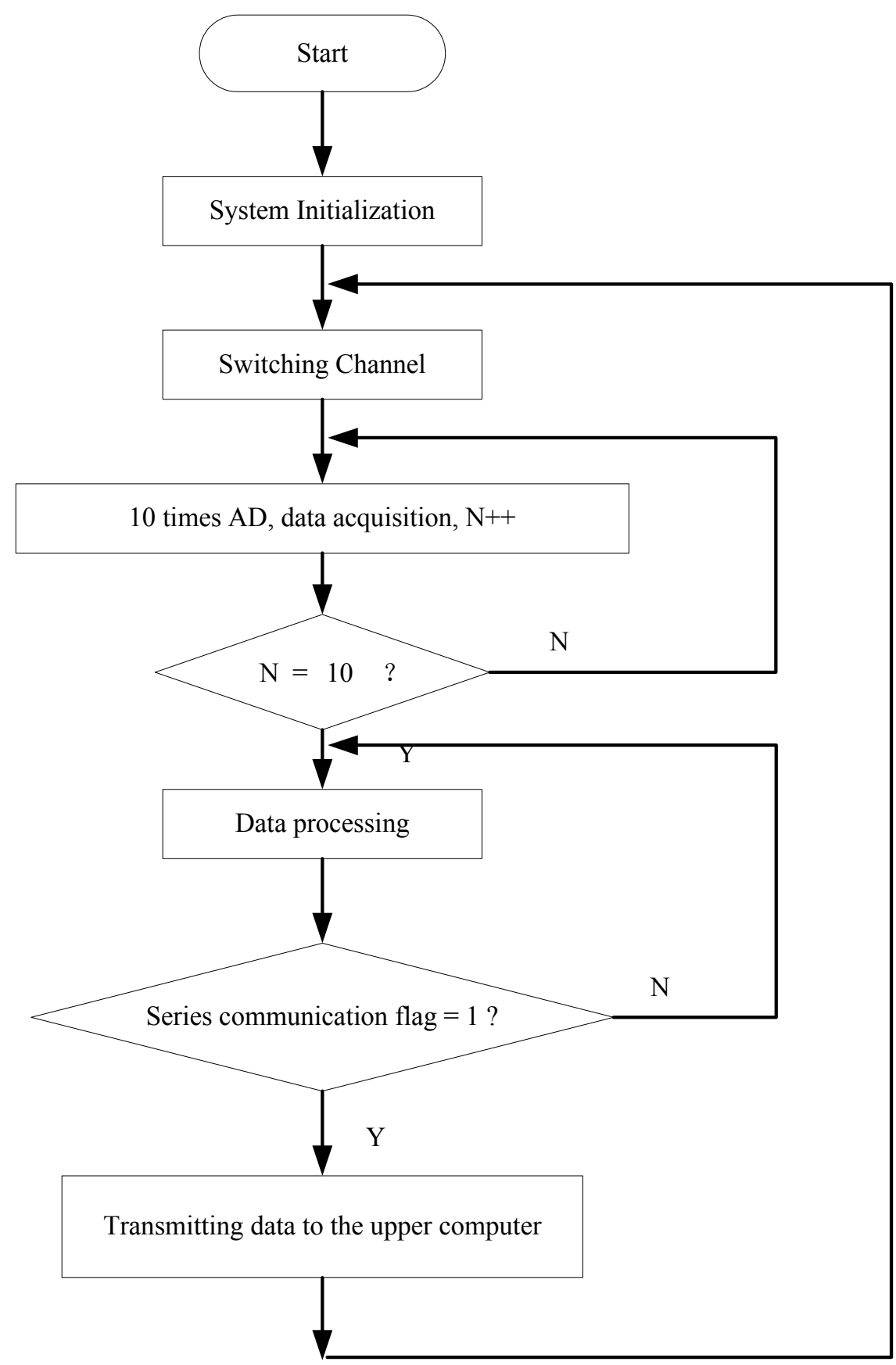

Figure 4. System Setting and Initialization Flow 


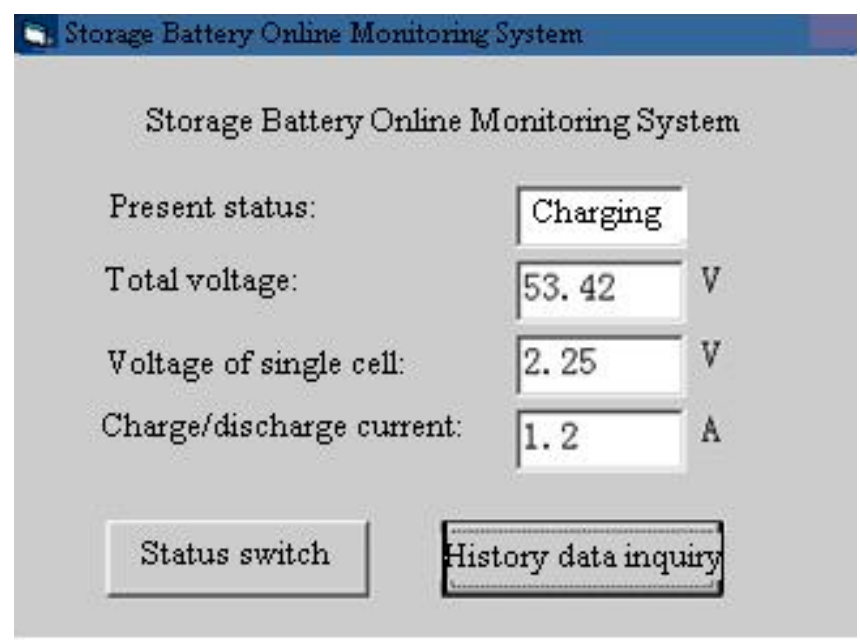

Figure 5. Interface of the Storage Battery Online Monitoring System 